\title{
Cosmic ray modulation by different types of solar wind disturbances
}

\author{
M. Dumbović ${ }^{1}$, B. Vršnak ${ }^{1}$, J. Čalogović ${ }^{1}$, and R. Župan ${ }^{2}$ \\ 1 Hvar Observatory, Faculty of Geodesy, Kačićeva 26, 10000 Zagreb, Croatia \\ e-mail: mdumbovic@geof.hr \\ 2 Institute of Cartography and Photogrammetry, Faculty of Geodesy, Kačićeva 26, 10000 Zagreb, Croatia
}

Received 15 July 2011 / Accepted 28 October 2011

\begin{abstract}
Context. Solar wind disturbances such as interplanetary coronal mass ejections (ICMEs) and corotating interaction regions (CIRs) cause short-term cosmic ray depressions, generally denoted as Forbush decreases.

Aims. We conduct a systematic statistical study of various aspects of Forbush decreases. The analysis provides empirical background for physical interpretations of short-term cosmic ray modulations.

Methods. Firstly, we analyzed the effects of different types of solar wind disturbances, and secondly, we focused on the phenomenon of over-recovery (the return of the cosmic ray count to a value higher than the pre-decrease level). The analysis is based on groundbased neutron monitor data and the solar wind data recorded by the Advanced Composition Explorer. The correlations between various cosmic ray depressions and solar wind parameters as well as their statistical significance are analyzed in detail. In addition, we performed a normalized superposed epoch analysis for depressions and magnetic field enhancements.

Results. The analysis revealed differences in the relationship between different solar wind disturbances and cosmic ray depression parameters. The amplitude of the depression for ICMEs was found to correlate well with the amplitudes of magnetic field strength and fluctuations, whereas for CIRs we found only the correlation between the amplitude of the depression and the solar wind disturbance dimension proxy $v t_{B}$. Similar behavior was found for shock and no-shock events, respectively. The CIR/ICME composites show a specific behavior that is a mixture of both ICMEs and CIRs. For all analyzed categories we found that the duration of the depression correlates with the duration of the solar wind disturbance. The analysis of the over-recovery showed that there is no straightforward relationship to either "branching-effect" or geomagnetic effects, therefore we propose a scenario where the "branching-effect" is caused by several factors and is only indirectly related to the over-recovery.
\end{abstract}

Key words. Sun: coronal mass ejections (CMEs) - solar-terrestrial relations - cosmic rays

\section{Introduction}

Disturbances in solar wind (SW) parameters such as proton speed, density and temperature, accompanied by highly fluctuating compressions of interplanetary magnetic field (IMF) cause short-term depressions in the galactic cosmic ray (CR) flux. Generally, these depressions are denoted as Forbush decreases (FDs), since they were first reported by Forbush (1937). Forbush decreases were related to solar activity by Simpson (1954). Depending on the nature and origin of the solar wind disturbances (SWDs), they can be classified as interplanetary coronal mass ejections (ICMEs) and corotating interaction regions (CIRs). The ICMEs are the interplanetary counterparts of coronal mass ejections, i.e., powerful eruptions of coronal magnetoplasma traveling through the heliosphere, whereas CIRs are related to the solar-wind high-speed streams originating in lowlatitude coronal holes. Because the pattern of the SW and IMF disturbances is different for these two phenomena, they can be readily identified in the in-situ measurements (see e.g. Iucci et al. 1979; Burlaga et al. 1984; Cane 2000; Richardson 2004). With respect to this, we note that interaction of these two phenomena leads to the occurrence of complex events (Belov et al. 2001) that represent a separate class of SWDs.

There are observational indications that mechanisms by which different types of SWDs produce CR depressions are different. Previous studies have shown that ICMEs produce sporadic strongly asymmetric depressions, while CIRs produce recurrent, more symmetric and relatively shallow depressions (for a comprehensive overview of short-term depressions in the CR count see Lockwood 1971; Cane 2000; Richardson 2004). Furthermore, the depressions tend to be deeper when a shock is formed at the leading edge of the disturbance (e.g. Badruddin et al. 1986; Singh \& Badruddin 2007), producing a two-step decrease (so-called classical FD, see e.g. Cane 2000).

This indicates that the reduced diffusion plays an important role in decreasing the CR flux, because the shock-sheath region is characterized by a highly fluctuating magnetic field, which reduces the $\mathrm{CR}$ diffusion length. Indeed, most of the theoretical models for transport of CRs emphasize the importance of pitch angle scatter and the magnetic field fluctuations, both for galactic cosmic rays (based on the Parkers transport equation, see e.g. Le Roux \& Potgieter 1991; Kota \& Jokipii 1991; Richardson et al. 1996; Potgieter 1998, and references therein) and also solar cosmic rays (based on the Roelof equation of focused transport, see e.g. Dröge 2000; Verkhoglyadova et al. 2010, and references therein). Along with diffusion, CRs are modulated by several other effects: convection by solar wind, gradient and curvature drift, and energy loss (Parker 1965), where the last one is usually considered as negligible for high-energy particles $(E>1 \mathrm{GeV}$, e.g. Le Roux \& Potgieter 1991). 
Regarding the two-step FDs caused by ICMEs, the reduceddiffusion model is thought to be appropriate for the shock-sheath region, whereas the exclusion of CRs because of the ejection itself is suspected to be caused by their closed magnetic field structure (Wibberenz et al. 1998). Two-step FDs are in the focus of several recent studies, especially those that are associated with magnetic clouds (e.g. Yu et al. 2010). They are not only the most prominent events, showing highest depression amplitudes (Cane 2000), they are also found to be useful in unrevealing the internal structure and geometry of ICMEs (e.g. Kuwabara et al. 2009; Richardson \& Cane 2011).

From the observational point of view, the influence of ICMEs and CIRs on CR modulation was previously investigated through a number of studies employing the superposed epoch analysis (e.g. Iucci et al. 1979; Badruddin et al. 1986; Badruddin 1996; Singh \& Badruddin 2007), statistical analysis (e.g. Cane et al. 1996; Richardson et al. 1996; Belov et al. 2001; Čalogović et al. 2009; Chilingarian \& Bostanjyan 2010) and case-studies (e.g. Jämsén et al. 2007). The results were frequently mutually conflicting, and consequently did not provide a clear empirical bacground. The correlation between the depression amplitude and some CIR parameters was found by Richardson et al. (1996) and Čalogović et al. (2009). Richardson et al. (1996) pointed out that the SWD speed might be the most important parameter, favoring a diffusion-convection model for this behavior. On the other hand, Čalogović et al. (2009) found a statistically significant correlation between the depression amplitude and the SWD magnetic field strength, which is more in favor of the diffusiondrift model proposed by Kota \& Jokipii (1991). Similar inconsistencies were found in studies of ICMEs. For example, Cane et al. (1996) declared speed as a poor predictor of depression amplitude, whereas Chilingarian \& Bostanjyan (2010) found a strong correlation between the two. Another aspect of CR modulation, the time profile, was found to be related to the speed for both CIRs (Iucci et al. 1979) and ICMEs (Badruddin et al. 1986; Penna \& Quillen 2005), but some questions still remain open (see, e.g. Lockwood et al. 1986; Jämsén et al. 2007).

In our previous study (Dumbović et al. 2011, hereafter Paper I) we examined the relationship between various SWD parameters and FD characteristics without distinguishing different types of SWDs. In this paper the sample of events used in Paper I is divided into subsamples according to SWD types, and the results are compared with those obtained in Paper I for the whole population, i.e. SWDs in general. We also consider in more detail the "branching effect" noticed in Paper I as well as the role of geomagnetic effects and the phenomenon of over-recovery (i.e., the return of the $\mathrm{CR}$ count to values above the pre-decrease level).

\section{Data and method}

A total of 26 periods between 1998 and 2005, each covering intervals of 20 days, were selected using the list of identified CME-ICME pairs prepared by Schwenn et al. (2005) and the case-study list of the European FP7-project SOTERIA (http: // soteria-space.eu/). To eliminate the daily variations, an average cosmic ray count rate (CR count) of three to four neutron monitor (NM) stations of similar rigidity located at different longitudes (see Appendix A of Paper I) was calculated, using hourly averaged data corrected for atmospheric pressure. The NM recordings were taken from the Space Physics Interactive Data Resource (SPIDR; http://spidr.ngdc.noaa.gov/spidr/). We used in-situ solar wind data from the Advanced Composition
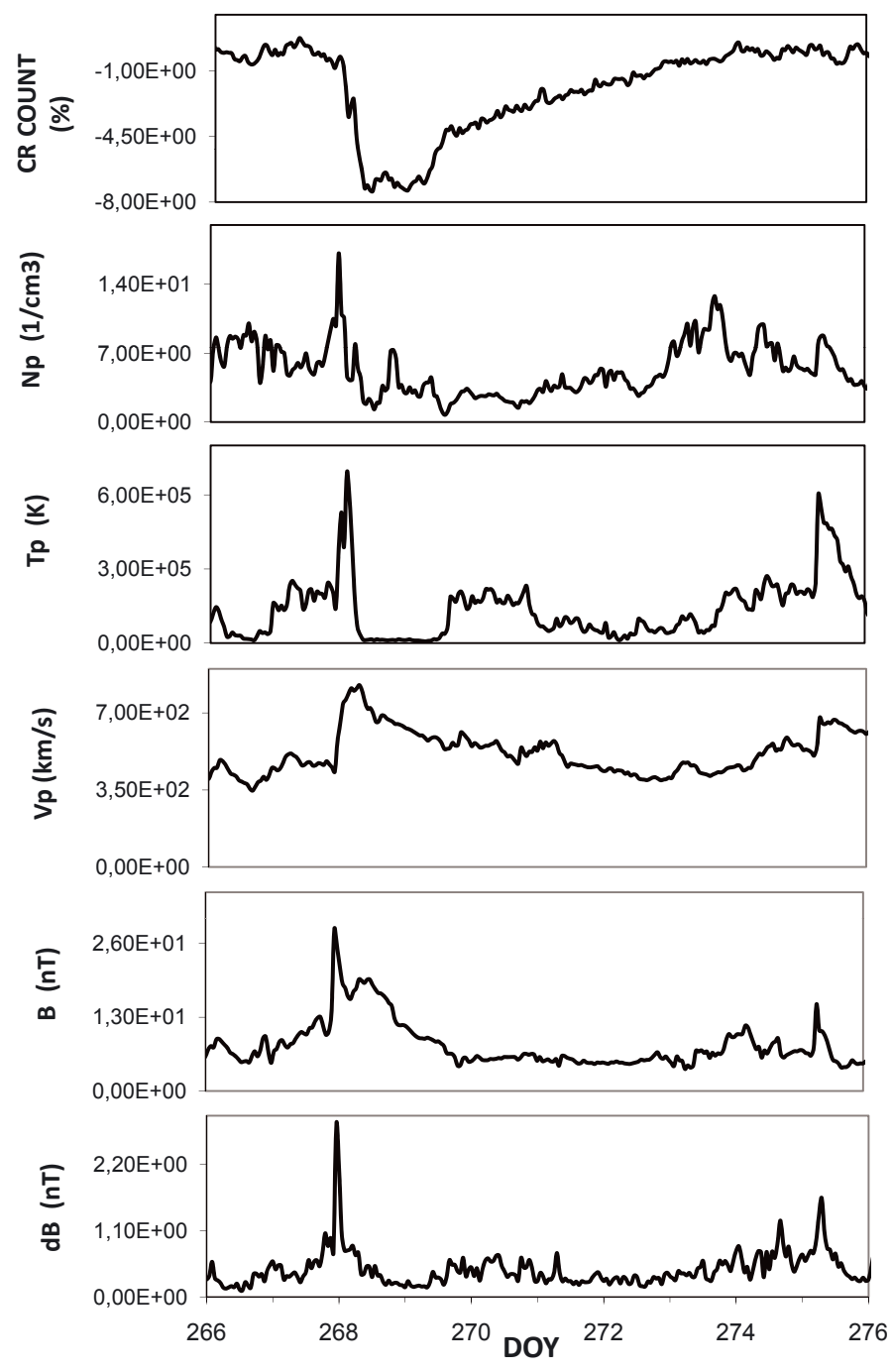

Fig. 1. ICME that arrived on 24/25 September $1998($ DOY $=268 / 269)$. The simultaneous step-increase in all the parameters is a signature of the ICME-driven shock, whereas the consecutive low-temperature region is specific for the ejection itself (e.g., Cane 2000). The CR count returns to the pre-decrease value, i.e., there is no over-recovery.

Explorer (ACE; Stone et al. 1998) database for SWD parameters. In particular, Level-2 data from the Solar Wind Electron, Proton, and Alpha Monitor (SWEPAM; McComas et al. 1998) and the magnetometer instrument (MAG; Smith et al. 1998) were analyzed. The SWDs were identified as increases in solar wind speed and interplanetary magnetic field (IMF) strength and fluctuations, and were associated with a decrease in the CR count (Forbush decrease, FD). The characteristics of the identified SWDs and the corresponding FDs were then measured as described in Fig. 1 of Paper I. A list of events, a detailed description of data handling and the measurement procedure as well as the list of measured parameters are given in Paper I.

The measured quantities involve the amplitude of magnetic field enhancement $(B)$, amplitude of the magnetic field fluctuations $(\delta B)$, relative increase in solar wind speed $\left(v_{\text {rel }}\right)$, maximum solar wind speed associated with the disturbance $\left(v_{\max }\right)$, duration of the disturbance (measured as the duration of the enhancement in the magnetic field strength, $t_{B}$ ), amplitude of the CR depression (hereafter, $|F D|)$ and the duration of the depression $\left(t_{\mathrm{FD}}\right)$ (see Fig. 1 in Paper I). Using these quantities, several combined parameters were employed as proxies of physical quantities (for 
M. Dumbović et al.: Cosmic ray modulation by different types of solar wind disturbances
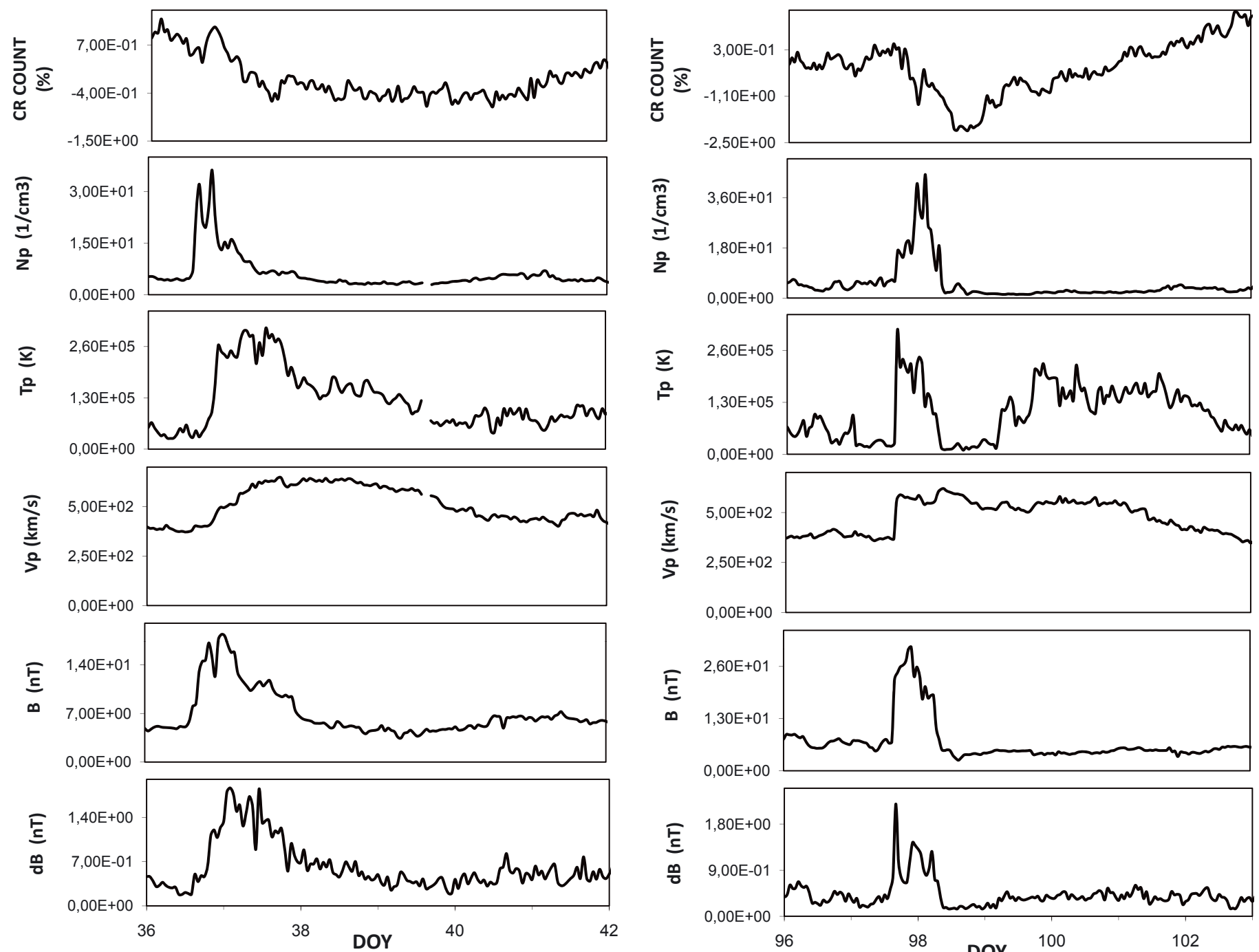

Fig. 2. CIR that occurred on 5 February $2000(D O Y=36)$. The density and magnetic field peak at the forehead of the high-speed stream, whereas the increase in speed and temperature coincides with the decrease of density. The CR count in the recovery phase does not exceed the pre-decrease value, i.e., there is no over-recovery.

details see Paper I): $B t_{B}$ as the proxy for the time integral of the IMF perturbation (measure of the "strength" of the disturbance), the $B v$ product as the proxy for maximum (convective) electric field (can also be regarded as the unit-width magnetic flux that passes over the observer in unit time), $B v t_{B}$ as the proxy for the magnetic flux (per unit-width of the disturbance cross-section), and $|F D| t_{\mathrm{FD}}$ as a measure of the total amount of CRs reduced by the passage of the disturbance.

First, the data set was sorted by the type of SWD, i.e., a distinction was made between interplanetary coronal mass ejections (ICME), corotating interaction regions (CIR), and mixed ICMECIR disturbances (mixed). The distinction between ICMEs and CIRs was made following Burlaga et al. (1984), Richardson et al. (1996), Cane (2000) and Richardson (2004), whereas the events that showed characteristics of both were denoted as mixed events. Examples of ICME-, CIR-, and mixed-events are presented in Figs. 1-3, respectively.

In the next step, the events were separated from those with and without a shock. The disturbances that propagate with supersonic speed produce a magnetohydrodynamic shock at their

front, where the shock-sheath region is characterized by high density, temperature and magnetic field, and by the enhanced level of the magnetic field fluctuations (see, e.g. Burlaga et al. 1984; Cane 2000). Differences between the shock-associated and no-shock events were studied by Badruddin et al. (1986) and Badruddin (1996). However, they applied only the superposed epoch analysis, lacking quantitative statistical results. Figures 1 and 3 illustrate events associated with a shock. In both cases the discontinuities are associated with a two-step fall of CR count, which is typical for shock-related disturbances (see, e.g., Wibberenz et al. 1998; Cane 2000). The discontinuity is absent in Fig. 2, which illustrates a typical no-shock event.

Finally, the events were divided into those showing the over-recovery and those without it. The phenomenon of overrecovery, i.e., the situation where the $\mathrm{CR}$ count in the recovery phase exceeds the pre-decrease value, was noticed by Jämsén et al. (2007) in high-energy data measured by a muon detector. Though here we inspect only the low-energy NM data, 


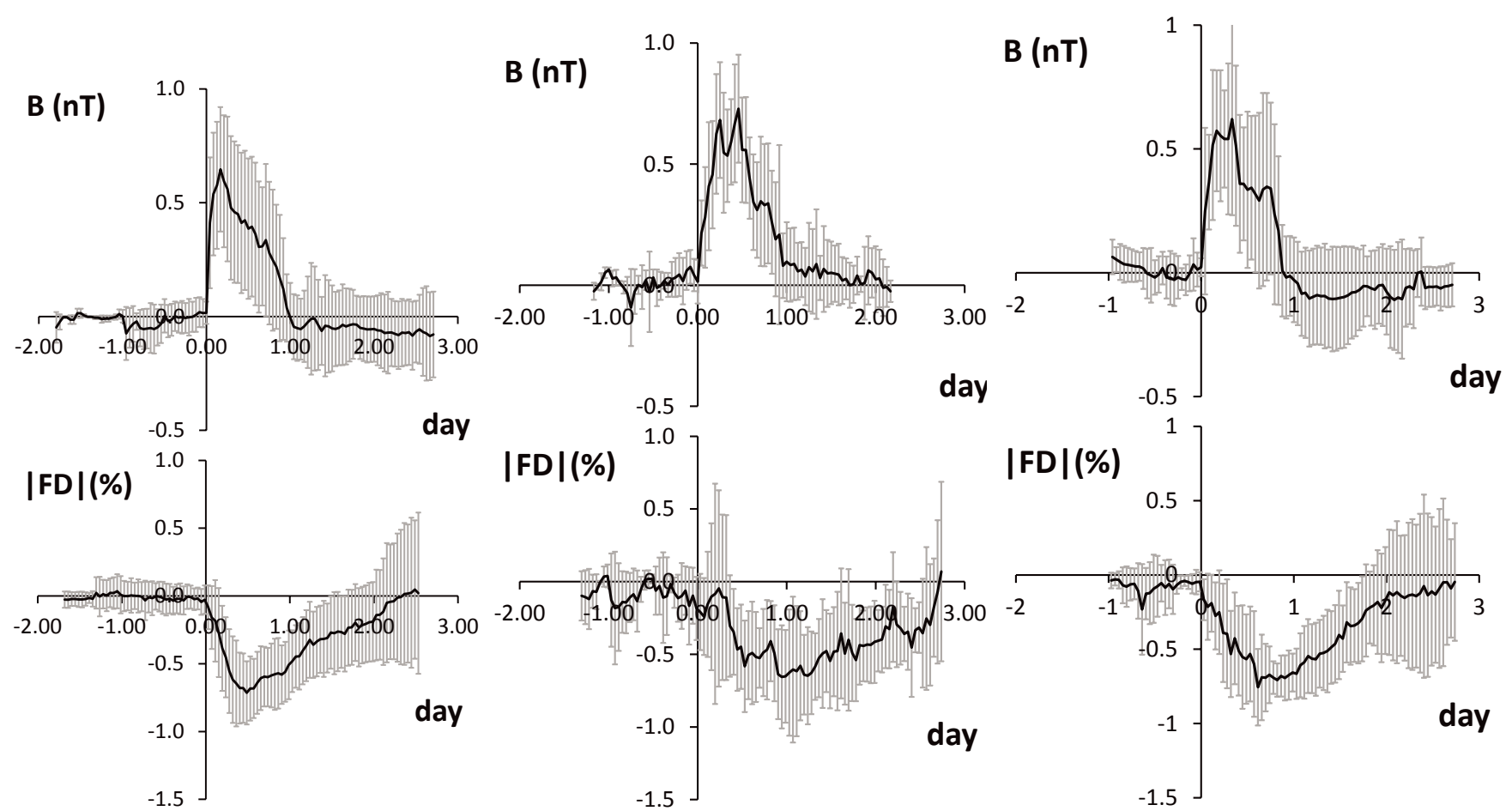

Fig. 4. Results of the superposed epoch analysis (FD magnitude, $|F D|$, and magnetic field enhancement, $B$ ) for different SWD types (left-ICMEs, middle-CIRs, right-mixed). Amplitudes in all events were normalized to 1 . The time scale was normalized to the duration of the SWD magnetic field enhancement. The SEA curves are drawn with solid black lines, whereas vertical gray lines represent standard deviations.

over-recovery is present in a considerable number of events (see example in Fig. 3).

In the following, we present the statistical analysis of correlations between various FD and SWD parameters, and the superposed epoch analysis (SEA) of the data. The latter is a statistical composing method, where a number of events are superposed to each other to reduce the noise, i.e. boost the signal. In the process, one obtains a statistical average of the events (for a detailed description of the procedure and its application in space research see e.g. Singh \& Badruddin 2006). The onset of the magnetic field enhancement was taken as the zeroepoch (i.e. the point of reference for superposition). The time scales of CR count and magnetic field strength were normalized to the duration of the SWD in question. Furthermore, for each event the values of the CR count and the magnetic field strength were normalized to $|F D|$ and $B$, respectively. In this way we avoided differences in amplitudes and durations of FDs, whereas the problems of the solar cycle variation effects are eliminated using relative CR counts (for a more detailed overview on the subject see Singh \& Badruddin 2006). This normalization procedure excluded FDs whose recovery is not well defined, those with an extreme over-recovery, and those with high fluctuations (i.e. events that deviated extremely from the rest of the sample) from the SEA sample. This was done because such events may lead to false statistical results, similar to those caused by outliers in a statistical correlation analysis. For the same reason, events whose type was ambiguous were excluded from the SEA. The SEA samples used in Sect. 3.1 for the comparison of different SWD types contain 33 ICMEs, 7 CIRs, and 9 mixed events, whereas the SEA comparison of shock and no-shock events (Sect. 3.2) includes 31 events that were associated with a shock and 13 events without a shock.

Two independent measurements were performed separately by M.D. and B.V. to reduce subjectiveness. The two data sets are denoted measurements-I and -II, respectively, and are fully presented in Appendices A and B of Paper I. Measurements-II dataset was used for the superposed epoch analysis (SEA) of CR and magnetic field data for different SWD types and shock/noshock events presented in Sects. 3.1 and 3.2.

\section{Results}

\subsection{Solar wind disturbances divided by type}

First, a normalized superposed epoch analysis was applied to different types of solar wind disturbances (Fig. 4). Note that all Forbush decrease curves were cut at the end of FD, i.e., at the point where CR count comes to the pre-event level.

Figure 4 clearly depicts differences between CIRs, ICMEs and mixed events in both $|F D|$ and $B$, in agreement with results presented by Badruddin et al. (1986); Badruddin (1996); Singh \& Badruddin (2007). In ICMEs the magnetic field strength increases sharply and returns to the pre-increase level fairly gradually. The corresponding FD has a sharp onset phase and gradual recovery. The CIRs show a more gradual increase in the IMF strength, and we also notice a more gradual decrease in FD, resulting in an almost symmetric form of the CR depression. The structure for mixed events is more complex, which is somewhat expected. The increase of the IMF has a sharp onset, similarly as in ICMEs, but the maximum value is shifted in time and there is another, clearly visible maximum associated with CIR. The corresponding depression is asymmetric in shape, though to a smaller degree than in ICMEs and there is an under-recovery, i.e., the $\mathrm{CR}$ count does not return to the pre-decrease value. These results show that the shape of the depression depends on the type/form of the magnetic field disturbance.

The onset of magnetic field enhancement and FD for ICMEs and mixed events is roughly simultaneous. In CIRs the situation 
Table 1. Correlation coefficients for the linear regression between FD and SWD parameters for different types of SWDs.

\begin{tabular}{|c|c|c|c|c|}
\hline \multicolumn{5}{|c|}{ a) $|F D|$} \\
\hline & $\begin{array}{c}\text { ICME } \\
\text { (43 events) }\end{array}$ & $\begin{array}{c}\text { CIR } \\
\text { (9 events) }\end{array}$ & $\begin{array}{c}\text { CIR }^{+} \\
\text {(20 events) }\end{array}$ & $\begin{array}{c}\text { mixed } \\
\text { (11 events) }\end{array}$ \\
\hline$B$ & 0.76 & $-0.04 * *$ & $0.04 * *$ & $0.86\left(0.06^{* *}\right)$ \\
\hline$\delta B$ & 0.75 & $-0.04 * *$ & $-0.03 * *$ & $0.68\left(0.11^{* *}\right)$ \\
\hline$v$ & 0.76 & $-0.13 * *$ & $0.04 * *$ & $0.39 * *$ \\
\hline$B t_{B}$ & 0.59 & $0.15 * *$ & $0.44 *$ & $0.87(0.22 * *)$ \\
\hline$B v$ & 0.69 & $-0.18 * *$ & $-0.04 * *$ & $0.36^{* *}$ \\
\hline$B v t_{B}$ & 0.71 & $0.02 * *$ & $0.37 * *$ & $0.39 * *$ \\
\hline$v t_{B}$ & 0.65 & $0.19 * *$ & 0.51 & $0.46^{* *}$ \\
\hline \multicolumn{5}{|c|}{ b) $t_{\mathrm{FD}}$} \\
\hline & $\begin{array}{c}\text { ICME } \\
\text { (26 events) }\end{array}$ & $\begin{array}{c}\text { CIR } \\
(5 \text { events })\end{array}$ & $\begin{array}{c}\text { CIR }^{+} \\
(17 \text { events })\end{array}$ & $\begin{array}{c}\text { mixed } \\
(11 \text { events })\end{array}$ \\
\hline$B$ & $0.32 *$ & $0.18 * *$ & $0.05^{* *}$ & $0.25^{* *}$ \\
\hline$t_{B}$ & 0.66 & $0.85^{*}$ & 0.84 & $0.48 * *$ \\
\hline$B t_{B}$ & 0.73 & $0.62 * *$ & 0.77 & $0.41 * *$ \\
\hline$B v$ & $0.38 *$ & $-0.03 * *$ & $-0.07 * *$ & $-0.09 * *$ \\
\hline$B v t_{B}$ & 0.66 & $0.30 * *$ & 0.63 & $0.05^{* *}$ \\
\hline$v t_{B}$ & 0.81 & $0.46^{* *}$ & 0.71 & $0.12 * *$ \\
\hline \multicolumn{5}{|c|}{ c) $|F D| t_{\mathrm{FD}}$} \\
\hline & $\begin{array}{c}\text { ICME } \\
\text { (26 events) }\end{array}$ & $\begin{array}{c}\text { CIR } \\
\text { (5 events) }\end{array}$ & $\begin{array}{c}\text { CIR }^{+} \\
(17 \text { events })\end{array}$ & $\begin{array}{c}\text { mixed } \\
(11 \text { events })\end{array}$ \\
\hline$B t_{B}$ & 0.79 & $0.63 * *$ & 0.75 & $0.89(0.54 * *)$ \\
\hline$\delta B t_{B}$ & 0.82 & $0.75^{*}$ & $0.48^{*}$ & $0.55 *(0.12 * *)$ \\
\hline$B v$ & 0.62 & $0.02 * *$ & $-0.10 * *$ & $0.13^{* *}$ \\
\hline$B v t_{B}$ & 0.79 & $0.35^{* *}$ & 0.65 & $0.24 * *$ \\
\hline$v t_{B}$ & 0.85 & $0.50 * *$ & 0.76 & $0.31 * *$ \\
\hline
\end{tabular}

Notes. Sample size is given in brackets. For other details see main text.

is not as clear because Fig. 4 indicates that the depression might start before the onset of the magnetic field enhancement. Because the effect is on the noise level, i.e., within the standard deviation range, and contradicts the results of the statistical analysis presented in Paper I, we have checked all individual cases and found no evidence for this behavior. Therefore, the effect should be considered as statistical artifact of SEA.

For ICMEs and mixed events the maximum of the magnetic field enhancement corresponds to the steepest part of the depression, between the FD onset and minimum. In case of CIRs this is unclear, because of noise. The FD reaches minimum before the end of the magnetic field enhancement in ICMEs, whereas for CIRs the two are approximately contemporaneous, and for the mixed events the correspondence between the two is unclear because of the existence of the secon maximum in the magnetic field enhancement.

In the next step we investigate differences in characteristics of FDs associated with ICMEs, CIRs, and mixed events through detailed analysis of correlations between different FD parameters (magnitude $|F D|$, duration $t_{\mathrm{FD}}$, and the product $|F D| t_{\mathrm{FD}}$ ) and SWD characteristics $\left(B, \delta B, v, t_{B}\right.$, and the products $B t_{B}, \delta B t_{B}$, $\left.B v, B v t_{B}, v t_{B}\right)$ applied to each SWD type separately. The correlation coefficients for all correlations are presented in Table 1, whereas the most interesting results are presented in Fig. 5. Correlations whose $t$-test significance level is in the range $0.05-$ 0.1 (i.e., $90-95 \%$ probability that the correlation is not accidental) are marked by an asterisk, whereas a double asterisk marks statistically insignificant correlations. Unmarked values are statistically significant at a 0.05 level or better.

Since the samples used for the correlation analysis are small (e.g., there are only 9 CIRs), the reliability of the correlation coefficients and the $t$-test could become questionable.
Therefore, we additionally applied a Monte Carlo method of checking the statistical significance, based on the calculation of the correlation coefficient from random samples (for details see Appendix A). The Monte Carlo method confirmed the significance levels obtained by $t$-test. It also revealed an outlier in the sample of mixed events, a peculiar event of 6 November 2001, which had a much more enhanced magnetic field than other mixed events.

Panels a, b, and c in Table 1 represent correlations between various SWD parameters and FD magnitude $(|F D|)$, duration of $\mathrm{FD}\left(t_{\mathrm{FD}}\right)$, and the product $|F D| t_{\mathrm{FD}}$, respectively. The sample where our measurements-I CIR-dataset was supplemented by the events analyzed by Čalogović et al. (2009) is denoted as $\mathrm{CIR}^{+}$(for explanation see text). In the mixed-events column the results obtained by excluding the outlier are given in brackets. Note that the outlier is not present in the parameters involving SWD speed and duration, because of lack of data for this particular event.

A two-sample $t$-test was applied to ICME/CIR, CIR/mixed and ICME/mixed samples to see if there is a difference in the mean values of $|F D|$. The results show that $|F D|$ caused by ICMEs are not significantly different from those caused by mixed events, whereas they are significantly higher than $|F D|$ values for CIRs. However, because ICMEs and mixed events are characterized also by stronger fields, the analysis was repeated considering only events with field strengths $B<20 \mathrm{nT}$ (to be compatible with CIR magnetic fields and also excluding the outlier in mixed events) and a similar result was obtained. The same conclusion can be drawn by inspecting Fig. 5. Therefore, we conclude that ICMEs and mixed events generally cause deeper depressions than CIRs, even if the IMF increase is the same for the two. This agrees with the results of the superposed epoch analysis in Badruddin et al. (1986) and Badruddin (1996). The same statistical procedure (a two-sample $t$-test) was applied for the magnetic field fluctuations where similar results were obtained. For $\delta B<2 \mathrm{nT}$ we find that mean $|F D|$ for CIRs is significantly lower than mean $|F D|$ for ICMEs. Again, no significant differences were found between ICMEs and mixed events.

In Table 1a the relationships between FD magnitude and various SWD parameters are presented. Clearly, the ICMEs show a significant positive correlation for each parameter listed, which is not the case for CIRs. This is illustrated for the magnetic field strength in Fig. 5a. For mixed events, $|F D|$ apparently correlates positively with the magnetic field strength and fluctuations, but this is only caused by an outlier (marked by black square). When the outlier is removed, the correlation disappears and there are also no correlations with other quantities (see the last column in Table 1). If we compare the outlier with the ICME sample, we find that it fits well into the ICME sample, showing that this event (6 November 2001) is strongly ICME-dominated.

Table $1 \mathrm{~b}$ shows correlation coefficients between the FD duration, $t_{\mathrm{FD}}$, and different $\mathrm{SWD}$ parameters for ICME, CIR and mixed events separately. No significant correlations are found at 0.05 level for mixed events and CIRs from our CIR-sample (2nd column). Significant positive correlations were found for ICMEs, except for the magnetic field strength, $B$, and the proxy for the maximum (convective) electric field, $B v$, where the correlation had a significance level of 0.1 . For the extended sample of CIRs ( 3 rd column, $\mathrm{CIR}^{+}$), where we also included CIRs from measurements I and Čalogović et al. (2009), the correlations improve and we see a similar pattern as for ICMEs.

The lack of significant correlation between $t_{\mathrm{FD}}$ and $B$ is different from the result obtained in Paper I for SWDs in general. This indicates that the positive correlation between $t_{\mathrm{FD}}$ and $B$ 

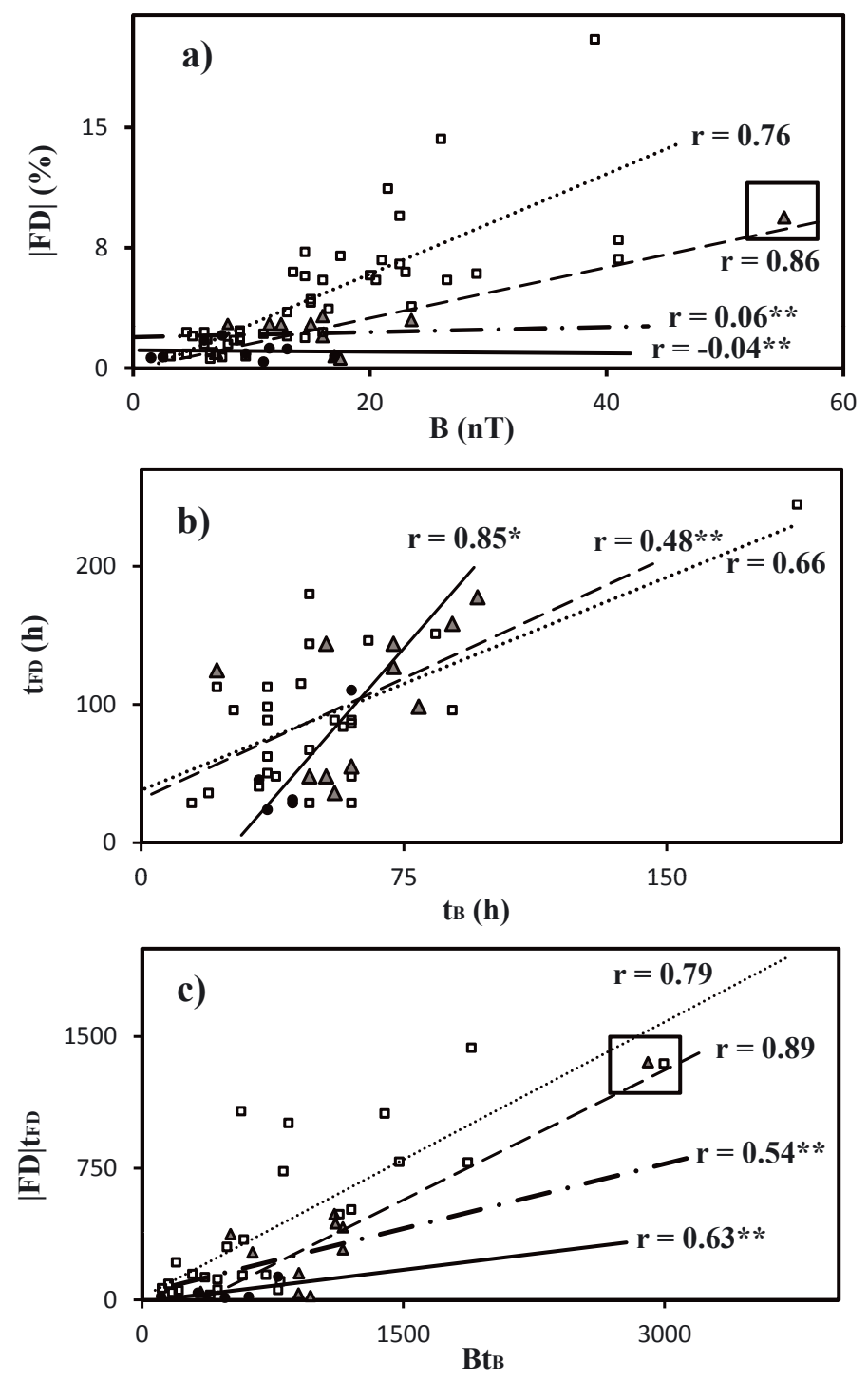

Fig. 5. Correlation of FD and SWD parameters for ICMEs (squares, dotted line), CIRs (black dots, solid line), mixed events (gray triangles, dashed line), and mixed events without outlier (circles, dashed-dotted line): a) FD magnitude, $|F D|$, versus magnetic field enhancement, $B$ ); b) FD duration, $t_{\mathrm{FD}}$, versus SWD duration, $\left.t_{B}\right)$; c) combined parameters. Correlation coefficients marked by an asterisk denote significance level of $0.05-0.1$, whereas a double asterisk denotes statistically insignificant values. The outlier (6 Nov. 2001) is marked by a black square in Figs. a) and $\mathbf{c}$.

obtained for SWDs in Paper I might be a consequence of some third parameter that influences both $B$ and $t_{\mathrm{FD}}$, i.e., that there is really no direct link between these two. The SWD speed might be this parameter because it is related to the duration of SWD (it is the interval during which the SWD passes the observer) and is also correlated with $B$ for ICMEs $(r=0.81)$.

Table 1c presents the correlations of various combined SWD-parameters and the combined parameter $|F D| t_{\mathrm{FD}}$, whose physical meaning is explained in Sect. 2 and in Paper I. Table 1c and the example presented in Fig. 5c show that somewhat better correlations are obtained for all types of SWDs when combined parameters are employed than for simple parameters (Figs. 5a and b). This is especially evident for ICMEs and agrees with results for SWDs in general (Paper I). This confirms the conclusion reached in Paper I, that combined parameters represent relevant physical quantities themselves, and that these quantities should be included in theoretical modeling. This is also true for mixed events, although the results are very much influenced by an outlier. Without the outlier, the statistical analysis shows a more CIR-like behavior, whereas with the outlier the behavior is more ICME-like. This leads to the conclusion that some mixed events will be ICME-dominated and some will be CIR-dominated, depending on the characteristics of the event. This can lead to contradictory results, as documented by the presented consideration of the outlier effect, i.e., one should be cautious when dealing with mixed events.

Finally, we consider the results of the CIRs more closely. The 2 nd column of Table 1 shows that there are no statistically significant correlations between $|F D|$ and any of the CIR parameters listed, which disagrees with previous studies (see e.g. Richardson et al. 1996; Čalogović et al. 2009). However, our CIR-sample is quite small (nine events) and the events are grouped in a relatively narrow interval of magnetic field strengths, as can be seen in Fig. 5a. To enlarge our sample we supplemented it by the events studied by Čalogović et al. (2009). In this way, the CIR-sample was enlarged to 20 events. Measurements of the SW magnetic field strength and speed were taken from Čalogović et al. (2009), whereas other quantities were re-measured to be consistent with measurementsII dataset. Because the maximum SW speed was measured in Čalogović et al. (2009), a relative value was approximated using the quiet-time $\mathrm{SW}$ speed value at $1 \mathrm{AU} \sim 400 \mathrm{~km} \mathrm{~s}^{-1}\left(v_{\text {rel }}=\right.$ $v_{\max }-400 \mathrm{~km} \mathrm{~s}^{-1}$ ). Though Čalogović et al. (2009) also measured $|F D|$, their data reduction was somewhat different (e.g., different NM stations were used) and we re-measured the CR data in the same way as in the measurements-II data. The results are presented in 3rd column of Table $1\left(\mathrm{CIR}^{+}\right)$. Evidently, they are more or less similar (though correlation coefficients increased somewhat) to the results obtained for measurements-II dataset (2nd column), except for correlations that include the SWD duration, which became statistically significant. In this respect we note that the supplemented events occurred in the declining phase of the solar cycle, when the CIR-related activity was not greatly influenced by ICMEs (Vršnak et al. 2007a).

\subsection{Shock-associated and no-shock events}

Shock-associated and no-shock events were also separated. In some cases it was unclear whether or not the shock was present (e.g., possible low-amplitude shock within noisy data), so these were excluded from the statistical analysis.

The majority of SWDs in the employed sample were found to be associated with a shock (79\% in measurements-I and $59 \%$ in measurements-II). Again, the correlation of FD characteristics $\left(|F D|, t_{\mathrm{FD}}\right.$ and $\left.|F D| t_{\mathrm{FD}}\right)$ with SWD parameters $(B, \delta B, v$, and $\left.t_{B}\right)$ and combined SWD parameters $\left(B t_{B}, B v, B v t_{B}\right.$, and $\left.v t_{B}\right)$ were considered, but now separately for shock-associated and no-shock events. The results of the linear regression analysis are shown in Table 2 for two independent sets of measurements (measurements-I and -II) and are illustrated in Fig. 6 for the measurements-II data. The notation used in Table 2 and the organization of panels a, b, and c are the same as in Table 1. The same significance tests were used as in Sect. 3.1.

A positive correlation was found for shock-associated events between FD magnitude and all SWD parameters $\left(B, \delta B, v, B t_{B}\right.$, $B v, B v t_{B}$, and $v t_{B}$ ) for measurements-I and -II (see Table 2a). On the other hand, no-shock events mostly show no correlations to SWD parameters. The only exception is $v t_{B}$ product, where 
Table 2. Correlation coefficients for shock-associated and no-shock events.

\begin{tabular}{|c|c|c|c|c|}
\hline \multicolumn{5}{|c|}{ a) $|F D|$} \\
\hline & \multicolumn{2}{|c|}{ shock } & \multicolumn{2}{|c|}{ no-shock } \\
\hline & $\begin{array}{c}\mathrm{I} \\
\text { (44 events) }\end{array}$ & $\begin{array}{c}\text { II } \\
\text { (40 events) }\end{array}$ & $\begin{array}{c}\mathrm{I} \\
\text { (11 events) }\end{array}$ & $\begin{array}{c}\text { II } \\
(17 \text { events })\end{array}$ \\
\hline$B$ & 0.58 & 0.67 & $0.16^{* *}$ & $0.26^{* *}$ \\
\hline$\delta B$ & 0.76 & 0.73 & $-0.14 * *$ & $0.21 * *$ \\
\hline$v$ & 0.51 & 0.56 & $-0.10 * *$ & $0.23 * *$ \\
\hline$B t_{B}$ & 0.52 & 0.56 & $0.49 * *$ & $0.46^{*}$ \\
\hline$B v$ & 0.67 & 0.59 & $0.01 * *$ & $0.1 * *$ \\
\hline$B v t_{B}$ & 0.60 & 0.61 & $0.39 * *$ & $0.30 * *$ \\
\hline$v t_{B}$ & 0.40 & 0.47 & 0.65 & $0.47 *$ \\
\hline \multicolumn{5}{|c|}{ b) $t_{\mathrm{FD}}$} \\
\hline & \multicolumn{2}{|c|}{ shock } & \multicolumn{2}{|c|}{ no-shock } \\
\hline & $\begin{array}{c}\text { I } \\
\text { (44 events) }\end{array}$ & $\begin{array}{c}\text { II } \\
(30 \text { events })\end{array}$ & $\begin{array}{c}\mathrm{I} \\
\text { (11 events) }\end{array}$ & $\begin{array}{c}\text { II } \\
\text { (13 events) }\end{array}$ \\
\hline $\bar{B}$ & 0.43 & $0.27 * *$ & 0.59 & $0.08 * *$ \\
\hline$t_{B}$ & 0.54 & 0.66 & 0.69 & 0.86 \\
\hline$B t_{B}$ & 0.58 & 0.68 & 0.82 & 0.64 \\
\hline$B v$ & 0.44 & 0.30 & $0.31 * *$ & $-0.3 * *$ \\
\hline$B v t_{B}$ & 0.57 & 0.59 & 0.67 & $-0.04 * *$ \\
\hline$v t_{B}$ & 0.58 & 0.70 & 0.66 & $0.12 * *$ \\
\hline \multicolumn{5}{|c|}{ c) $|F D| t_{\mathrm{FD}}$} \\
\hline & \multicolumn{2}{|c|}{ shock } & \multicolumn{2}{|c|}{ no-shock } \\
\hline & $\begin{array}{c}\text { I } \\
\text { (44 events) }\end{array}$ & $\begin{array}{c}\text { II } \\
(30 \text { events })\end{array}$ & $\begin{array}{c}\mathrm{I} \\
(11 \text { events })\end{array}$ & $\begin{array}{c}\text { II } \\
\text { (13 events) }\end{array}$ \\
\hline$B t_{B}$ & 0.76 & 0.80 & 0.68 & 0.76 \\
\hline$\delta B t_{B}$ & 0.76 & 0.77 & $0.35 * *$ & $0.48 * *$ \\
\hline$B v$ & 0.68 & 0.54 & $0.05 * *$ & $-0.16^{* *}$ \\
\hline$B v t_{B}$ & 0.75 & 0.71 & $0.45 * *$ & $0.12 * *$ \\
\hline$v t_{B}$ & 0.59 & 0.65 & $0.55^{*}$ & $0.29 * *$ \\
\hline
\end{tabular}

Notes. Sample size is given in brackets. For other details see main text.

a positive correlation was found at a 0.05 significance level in measurements-I and 0.1 significance level in measurements-II. The highest correlation coefficient for shock-associated events is for the magnetic field fluctuations both in measurement-I and measurement-II. This is similar to the results obtained for SWDs in general (Paper I) and agrees with studies that emphasize post-shock turbulence as the main cause of the CR depression (e.g., Badruddin et al. 1986; Badruddin 1996), where increased fluctuations lead to $\mathrm{CR}$ reduction because of shortened diffusion length (see e.g. Wibberenz et al. 1998).

Figures $6 \mathrm{a}$ and $\mathrm{b}$ show the dependence of FD magnitude on magnetic field enhancement $(B)$ and fluctuations $(\delta B)$ for shock and no-shock events. Evidently, disturbances associated with shocks cause deeper depressions, as was reported by, e.g., Badruddin et al. (1986) and Singh \& Badruddin (2007). Shockrelated data points in Fig. 6a show "branching" as SWD data in general, noticed in Paper I. Events without shock show no such structure, but it should be noted that no-shock events generally have weaker field strengths and therefore are tied to the domain where the branching effect cannot be observed.

Generally, shock-associated events have stronger fields, higher field fluctuations and larger FD magnitudes than no-shock events. This can be seen in Figs. 6a and b. Inspecting the difference in scales of magnetic field enhancement and fluctuations between shock and no-shock events, one could reason that this might be the source of the difference in FD magnitude modulation. Accordingly, the difference between shock and no-shock events was checked by applying a two-sample $t$-test, where we excluded all shock-associated events with a field strength exceeding $17 \mathrm{nT}$. In this way we analyzed shock and no-shock
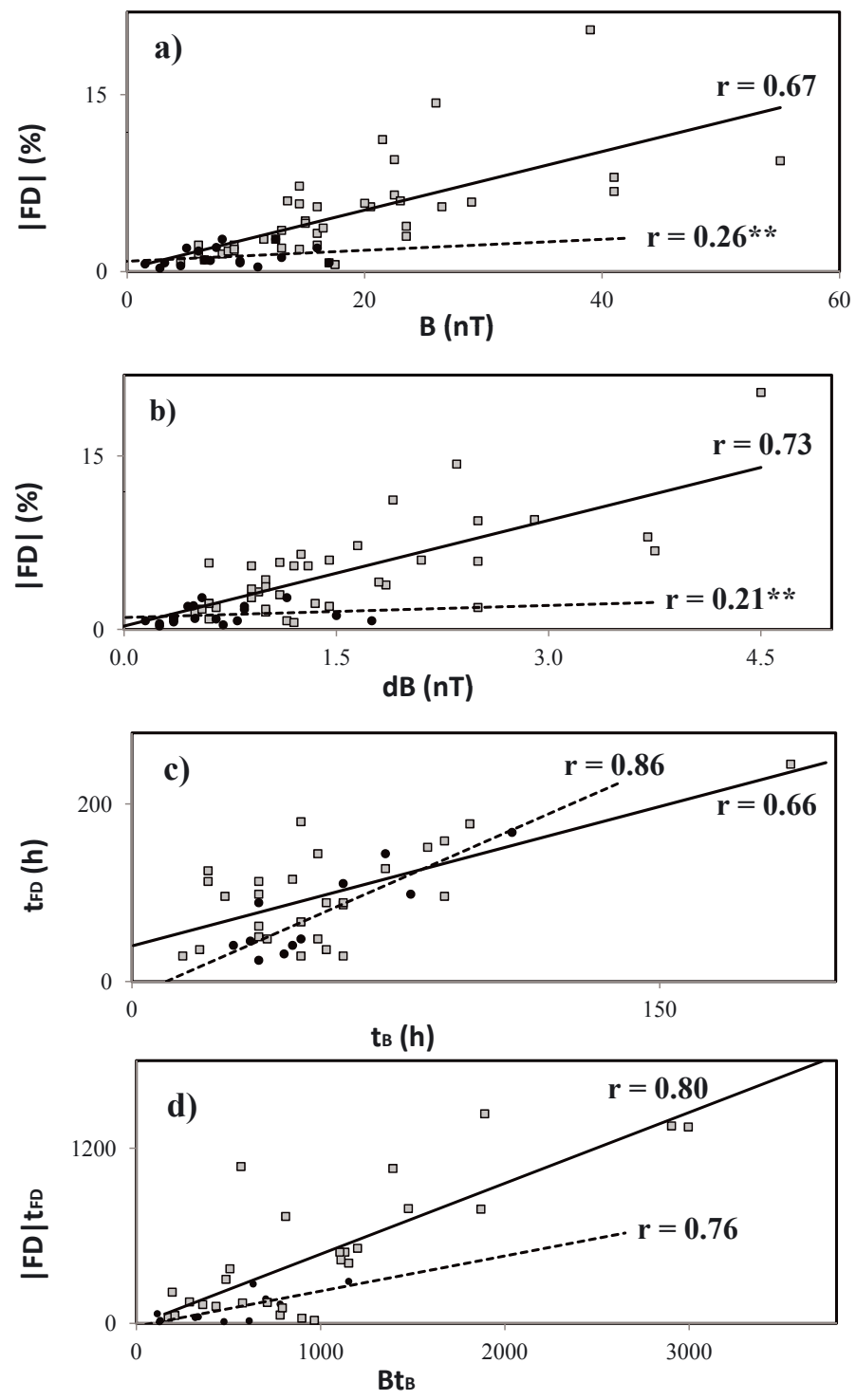

Fig. 6. Linear regression between different FD and SWD parameters for the shock-associated (squares, solid line) and no-shock (dots, dashed line) events: a) FD magnitude and magnetic field enhancement; b) FD magnitude and magnetic field fluctuations; c) FD duration and SWD duration; d) combined parameters. Double asterisk denotes statistically insignificant values. The results are from the measurements-II dataset.

events on the same scale of magnetic field enhancement. The test showed that for the same amplitude of magnetic field enhancement, no-shock SWDs cause FDs with a 55\% smaller magnitude compared to FDs caused by SWDs with shock (in measurements-I and -II).

In addition, a linear regression analysis was applied to the shock-associated data for small fields only $(B<17 \mathrm{nT})$, and again a significant positive correlation was found $(r=0.58$ and $r=0.56$ for measurements-I and -II, respectively). A similar analysis was performed regarding the magnetic field fluctuations with an analogous outcome. Therefore, for the same level of fluctuations we would still expect deeper depressions for shock SWDs as compared to no-shock SWDs.

The correlations between FD duration and different SWD parameters are presented in Table $2 \mathrm{~b}$ and Fig. 6c, where it can be seen that FD and SWD durations are correlated for shockassociated and no-shock disturbances. A two-sample $t$-test was 

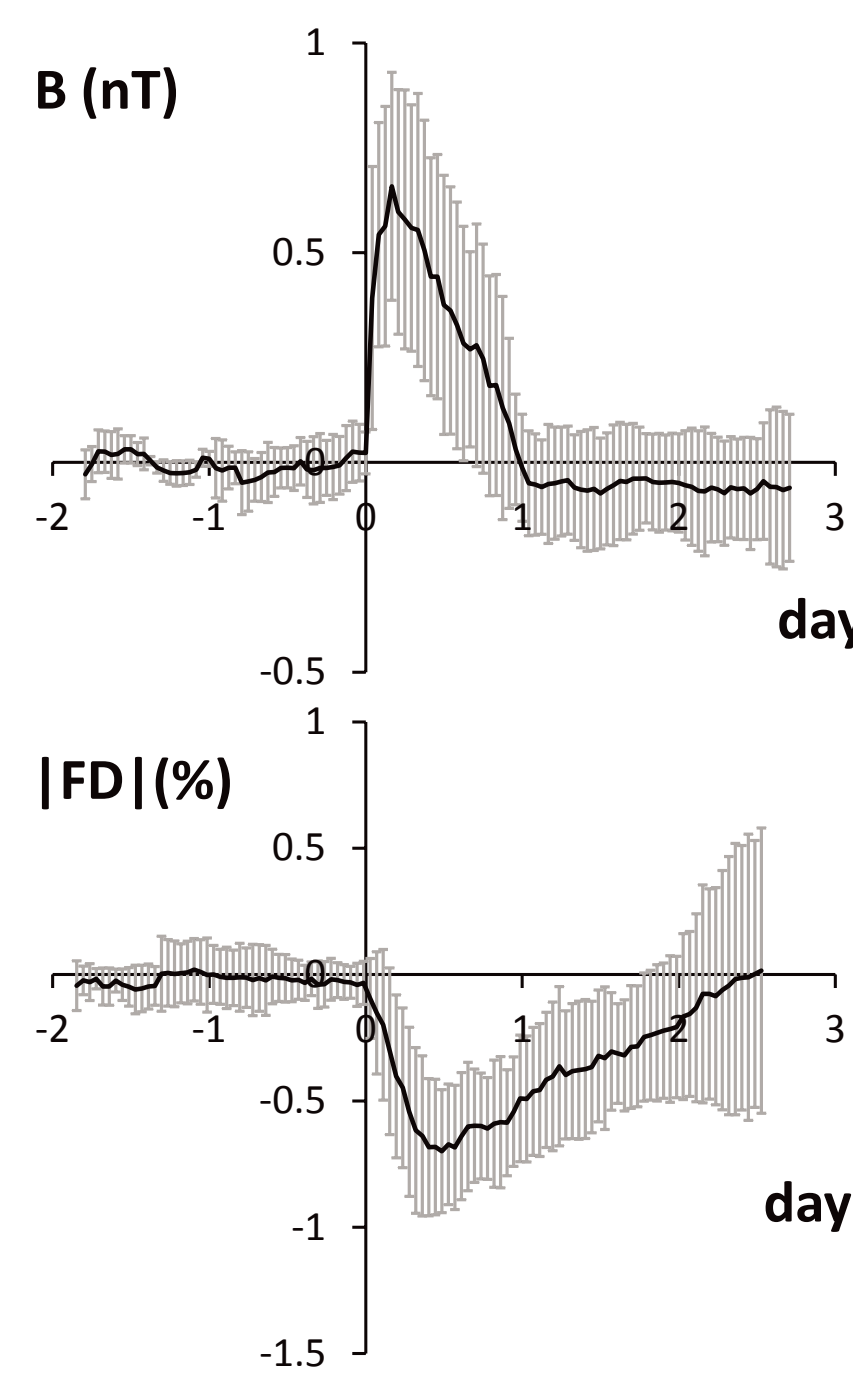

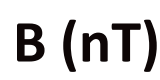

$-2$

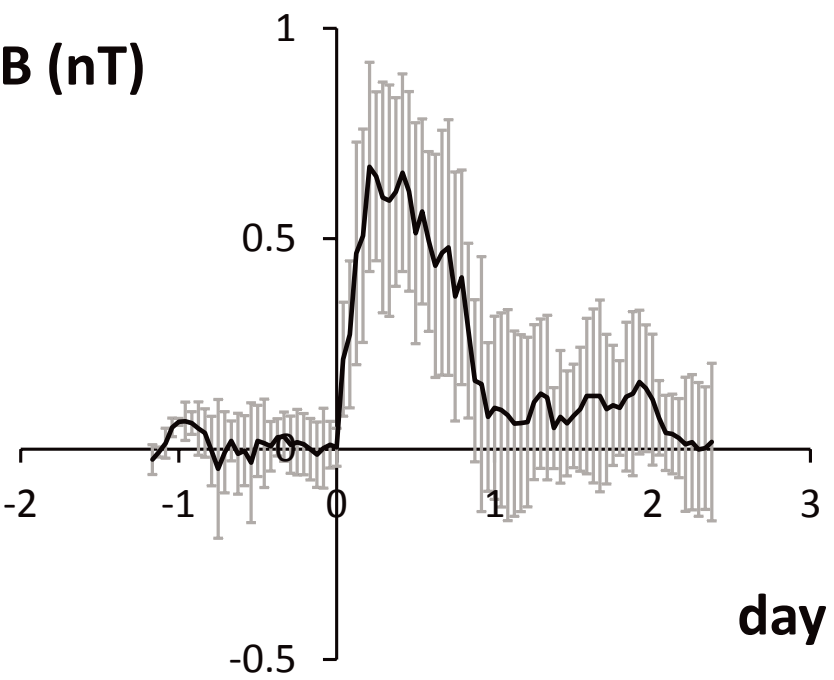

$|F D|(\%)$
1

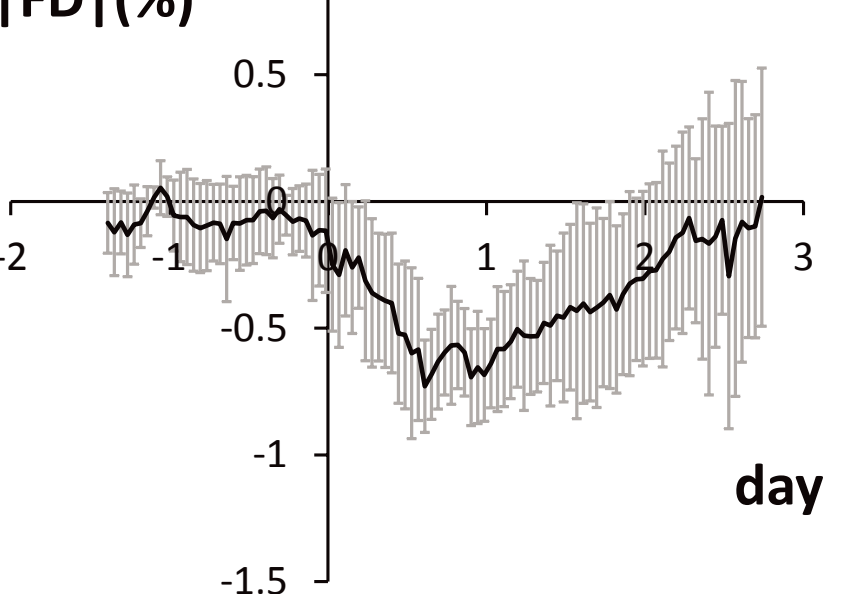

Fig. 7. Results of the superposed epoch analysis (FD magnitude, $|F D|$, and magnetic field enhancement, $B$ ) for shock (left) and no-shock events $($ right $)$. Amplitudes were normalized to 1 . The time scale was normalized to the duration of the SWD. The SEA curves are drawn by solid black lines, whereas vertical gray bars represent standard deviations.

applied, and no difference was found between the two, showing that a shock does not affect the duration of FD. The same behavior was noticed by Singh \& Badruddin (2007), who found that the duration of the recovery phase, and therefore the total duration, was influenced primarily by the high-speed stream, regardless of the association with shock. In addition, we found that for shock-associated events the duration of FD correlates with all SWD parameter products that involve $t_{B}$, where for events without shock only correlation with $B t_{B}$ product was found.

Finally, in Table $2 \mathrm{c}$ correlations concerning the product $|F D| t_{\mathrm{FD}}$ are presented. Shock-related events are better correlated with SWD parameters than $|F D|$ and $t_{\mathrm{FD}}$ separately, which is similar to the results obtained in Paper I for SWDs in general. For no-shock events, only a correlation with $B t_{B}$ was found, while for other products there is either no correlation or the results are inconclusive. Figure $6 \mathrm{~d}$ shows linear regression lines $|F D| t_{\mathrm{FD}}\left(B t_{B}\right)$ for shock and no-shock data, where two different trends can be seen. Shock-associated events have higher values of both FD and SWD parameter products. The difference was confirmed by a two-sample $t$-test for measurements-I and measurements-II in the same manner as for $|F D|(B)$ data.

Again, as with different types of SWDs, we employed a normalized superposed epoch analysis to inspect the differences in the relationship between the magnetic field enhancements and
FDs related to shock-associated and no-shock events. The results are presented in Fig. 7 and show that the averaged magnetic field enhancement has a sharp onset followed by a more gradual decay for SWDs associated with shock and the corresponding FD is highly asymmetric. The averaged magnetic field enhancement is more symmetric for no-shock events, resulting in a considerably more symmetric FDs. This outcome complements the results on SWD types presented in Sect. 3.1, confirming that the shape of the depression depends on the shape of the magnetic field enhancement. Again we find that the steepest part of FD corresponds to the maximum in $B$.

\subsection{Over-recovery, branching effect, and geoeffectiveness}

In some Forbush decreases the $\mathrm{CR}$ count returns to a higher level than it was before the FD onset. This phenomenon, called over-recovery, was noticed by Jämsén et al. (2007), but recorded by muon detector only in the most energetic CR data. Here we examine the over-recovery in the neutron monitor (NM) data, which, unlike muon detectors, also include lower energy CRs. The analysis was to a certain degree hindered if:

- the recovery phase was interrupted by another event; 
- the event starts by interrupting the recovery phase of another event;

- noise masks the pre-decrease level, or recovery phase of the event.

The first two effects are very important factors in examining the over-recovery because they can directly lead to erroneous identification of the effect, therefore these events were treated as a separate category. The full list of events and their classification is given in Paper I, where events were categorized as follows:

1. events that show no over-recovery and are not interrupted by another event (denoted $\mathrm{N}$ );

2. events that show no over-recovery, but are interrupted by another event (denoted $\mathrm{N}^{*}$ );

3 . events that show over-recovery, but start in the recovery phase of another event (denoted $\mathrm{Y}^{*}$ );

4. events that show over-recovery and do not start in the recovery phase of another event (denoted Y).

Out of 56 events, 36 (64\%) showed no over-recovery, where 18 of these $(32 \%)$ were of the category $\mathrm{N}^{*}$. The rest of 20 events $(36 \%)$ showed over-recovery, where 11 events $(20 \%)$ started in the recovery phase of another event (class $\mathrm{Y}^{*}$ ). When $|F D|$ is plotted against $B$ separately for events associated with overrecovery ( $\mathrm{Y}$ and $\mathrm{Y}^{*}$ events), and those not associated with it ( $\mathrm{N}$ and $\mathrm{N}^{*}$ events), an interesting correspondence is obtained between the two branches noticed in Paper I and these two samples (Fig. 8a). This indicates that the branching effect might be related to over-recovery. However, note that the event with the highest magnetic field strength that occurred 6 November 2001, does not fit into this scheme, i.e., the correspondence between branching and over-recovery/no-over-recovery samples in Fig. 8a is not complete. Finally, the branching effect might be linked to geomagnetic storms (GMSs), as noted by Chilingarian \& Bostanjyan (2010) and discussed in Paper I. This indicates that both the branching-effect and the over-recovery might be related to geomagnetic effects. Therefore an analysis was performed to see if these three phenomena are related.

A two-sample $t$-test for the data on events with and without over-recovery was applied to check if the mean values of $|F D|$ and $B$ for the two samples are different. The test showed no difference, unless only strong-field ICMEs are considered (Fig. 8b): when weak-field and non-ICME events are excluded (removing the "problematic" 6 November 2001 mixed event), we find a statistically significant difference in the mean $B$. Therefore, although indications that relate branching effect and over-recovery are found, the relationship, if exists, is certainly not a simple one.

To inspect effects of geomagnetic activity, the disturbance storm time (Dst) index was employed. It is a longitudinally averaged deviation from the quiet level of the horizontal geomagnetic field measured in the equatorial region (see, e.g., Koskinen \& Huttunen 2006; Vršnak et al. 2007b; Kane 2005, and references therein). The hourly averages of Dst were taken from the Kyoto Dst index service (http://wdc.kugi.kyoto-u. ac.jp/dstdir/index.html) in the same time-periods as for CR count and solar wind disturbance (SWD) parameters. Only Dst amplitudes with distinct depressions were considered meaning geomagnetic storms (GMSs). The Dst indices were categorized as in Kudela \& Brenkus (2004):

i) Dst $>-50 \mathrm{nT}$ (not geoeffective, type 0$)$;

ii) $-50>$ Dst $>-200 \mathrm{nT}$ (weakly geoeffective, type A);

iii) Dst $<-200 \mathrm{nT}$ (strongly geoeffective, type B).
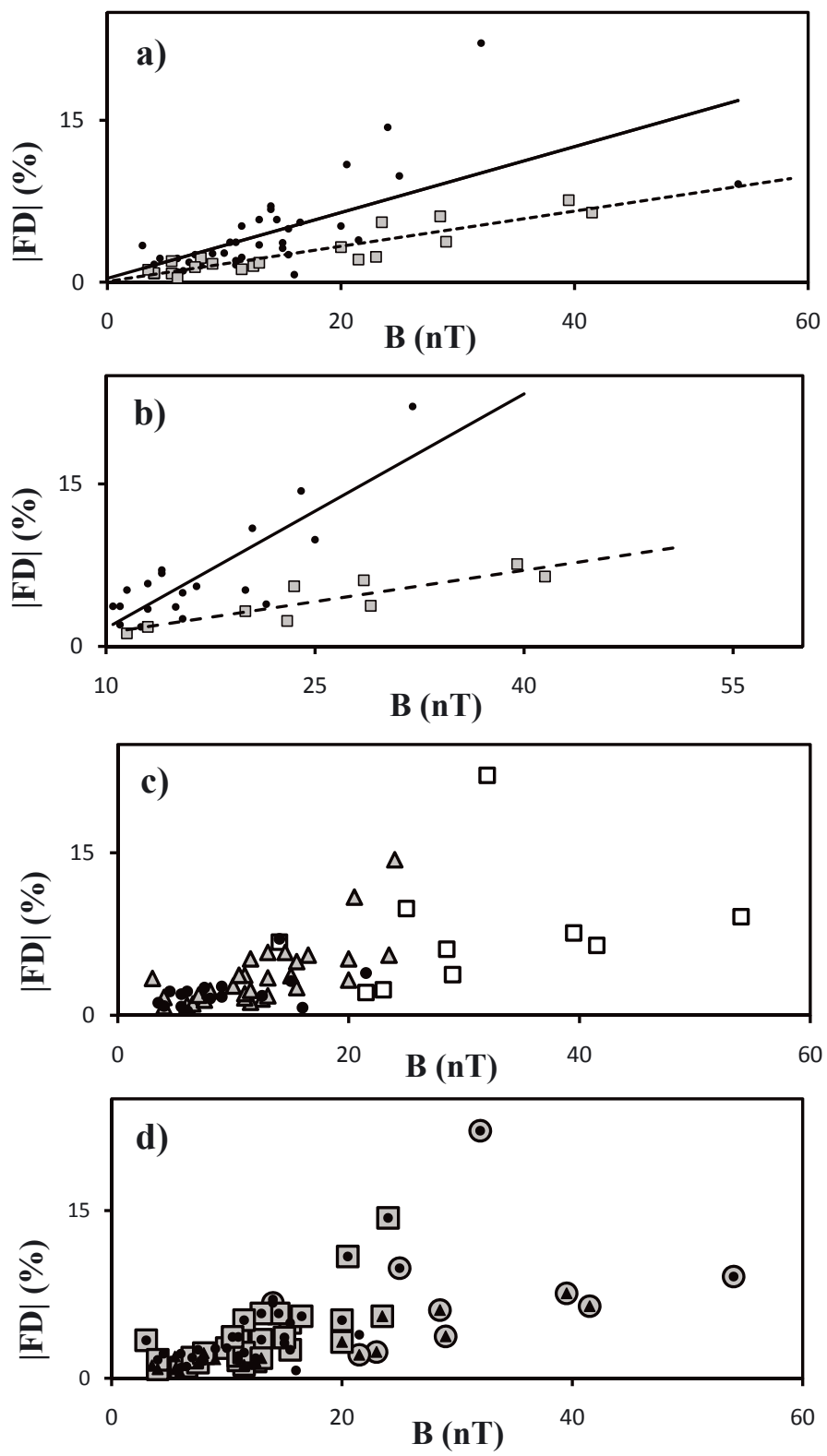

Fig. 8. Relationship between branching effect, over-recovery and geoeffectiveness as seen in $|F D|(B)$ plot: a) over-recovery (gray squares, dotted line) and no-over-recovery events (black dots, solid line); b) the same as in a) but only for ICMEs with field strength $B>10 \mathrm{nT}$; c) events associated with minor or no GMS ( $D$ st $>-50$, black dots), events associated with medium GMSs $(-50>D$ st $>-200$, gray triangles) and those associated with the strongest GMSs (Dst $<-200$, squares); d) a composite plot of events associated with GMSs in the range $-50>D$ st $>-200$ (squares), events associated with the strongest GMSs (Dst $<-200$, gray circles), over-recovery events (black triangles) and no-over-recovery events (black dots).

Similarly to the over-recovery phenomenon, there seems to be a relationship between the branching effect in $|F D|(B)$ data and geomagnetic activity: the lower branch is populated mainly by strongly geoeffective events (Fig. 8c). Two sample $t$-tests were applied to weakly and strongly geoeffective events to check if the mean values of $|F D|$ and $B$ differ between the two samples. The analysis showed that strongly geoeffective events tend to have stronger SWD fields associated with weaker FDs, which agrees 
with reduced rigidity cutoff scenario proposed by Chilingarian \& Bostanjyan (2009) and Chilingarian \& Bostanjyan (2010). However, Fig. 8c reveals that there are deviations; especially problematic is the event of 29 October 2003, which was associated with the strongest FD. Therefore, it seems that geoeffectiveness alone cannot account for the branching effect (although it might contribute). This behavior agrees with the findings of Kudela \& Brenkus (2004) that deep FDs are not necessarily associated with strong Dst perturbations. Similarily, we also investigated a possible relation between the over-recovery and ram pressure of the SWD, but we found none.

\section{Discussion and conclusion}

\subsection{Influence of different solar wind disturbances on cosmic ray depressions}

The superposed epoch analysis showed that for different shapes of magnetic field enhancement, associated with different solar wind disturbances, different shapes of depressions in cosmic ray count are to be expected. Furthermore, the shape of the depression (Forbush decrease) is related to the shape of the magnetic field enhancement. Apart from the shape, CR depressions caused by different SWDs also differ by magnitude, as we showed with a two-sample $t$-test in Sects. 3.1 and 3.2. A similar result was found by an SEA analysis in several studies (Badruddin et al. 1986; Badruddin 1996; Singh \& Badruddin 2007). For the same amplitude of the magnetic field enhancement and fluctuations, shallower depressions are expected for CIRs and no-shock events, as compared to ICMEs, mixed, and shock events, respectively.

The differences are also found in a linear regression analysis. The ICMEs and shock events were shown to have significant correlations between SW and IMF parameters and FD magnitude, whereas for CIRs and no-shock events we only found the correlation between the FD magnitude and disturbance width $\left(v t_{B}\right)$. The only feature that was common to all these SWDs (ICME, CIR, shock, no-shock) was the significant correlation between the duration of the depression $\left(t_{\mathrm{FD}}\right)$ and the duration of the SWD $\left(t_{B}\right)$, as well as the the correlation for the combined parameters $\left(|F D| t_{\mathrm{FD}}\left(B t_{B}\right)\right.$. It is interesting to note that the relationship between shock and no-shock events resembles the relationship between ICMEs and CIRs. This is visible in the linear analysis and the SEA results. Therefore, we inspected the relative numbers of CIRs in no-shock events and of ICMEs in shock events. In shock-associated events, $84 \%$ are ICMEs, and the rest are mixed events, meaning that shock-event sample and ICME sample are practically the same. On the other hand, no-shock sample includes $41 \%$ of CIR events, $18 \%$ (i.e. three events) could not be unambiguously identified as CIRs (denoted "CIR?" in the Paper I event list) and the rest are either ICMEs or mixed events. This implies that the no-shock sample and the CIR sample are different, i.e., there is a significant fraction of no-shock events that are ICME-related.

Therefore, considering all of the above mentioned, we conclude that CR modulation of ICMEs not associated with shock is similar to the CR modulation by CIRs, and that both are very different from the modulation by ICMEs associated with shock. This agrees with Wibberenz et al. (1998), who argue that the modulation in the shock-sheath region and in the ejecta are caused by different mechanisms and should be treated separately. Furthermore, the results of the linear regression indicate that the exclusion of CRs, for ICMEs with shock, is related to the magnetic field strength and fluctuations, which agrees with the propagating diffusive barrier scenario discussed in Wibberenz et al. (1998) and incorporated in model of Le Roux \& Potgieter (1991). We note that our result are based on measurements in which we did not separate the shock-sheath regions from the ejecta regions (for the study of the inner structure of the ICME and its influence on CRs see e.g. Richardson \& Cane 2011).

The lack of the correlation between FD and SWD parameters for CIRs is a different result from that obtained by, e.g., Richardson et al. (1996) and Čalogović et al. (2009). It opposes modeling based on the propagating region of the enhanced scattering and reduced drift (Kota \& Jokipii 1991) or enhanced convection (Richardson et al. 1996), since no correlations were found between the amplitude of the depression and magnetic field strength/fluctuations/speed. But the time profile of SWDs, which we found to be very important for CR exclusion in general, was not regarded within these models. As we showed here and in Paper I, combined parameters should be considered in future theoretical models. Secondly, although Monte Carlo tests were made, we still only considered a small sample and more importantly, the sample covers a relatively small range of $B$ and $|F D|$ compared to the accuracy of measurements.

Finally, by inspecting mixed events we found that they show a combination of ICME and CIR characteristics. When we excluded the ICME-dominated event that occurred on 6 November 2001 (the outlier), the statistical correlation analysis revealed that the mixed events show a more CIR-like behavior. In this respect, note that even if the mixed event sample without outlier is combined with a $\mathrm{CIR}^{+}$sample there is still no significant correlation between $|F D|$ and $B(r=0.25)$.

\subsection{The relationship between the "branching effect" and over-recovery}

When we combined the analysis of geoeffectiveness with overrecovery analysis, we obtained somewhat confusing results. In general it looks as though the over-recovery data and strong geomagnetic storms (GMSs) are following the lower branch of $|F D|(B)$, whereas data not associated with over-recovery generally coincide with weaker GMSs and follow the upper branch. However, there are deviations that cannot be disregarded, because the two extreme data points show a completely different behavior. The event of 6 November 2001, which has the highest field strength, was strongly geoeffective, showed no overrecovery and belonged to the lower branch of $|F D|(B)$. On the other hand, the deepest FD event, which occurred on 29 October 2003, also had no over-recovery and was strongly geoeffective, but was belonging to the upper branch of $|F D|(B)$ (Fig. 8d). This indicates that several parameters might combine to influence the branching effect, which could be related to both geoeffectiveness and over-recovery, but in a complex manner. Here we discuss possible causes of the branching effect and propose a possible scenario that can lead to it.

First, there is one more aspect of the branching effect that has to be considered in the analysis, namely that branching is lost when combined parameters are correlated $\left(|F D| t_{\mathrm{FD}}\right.$ vs. $B t_{B}$, see Paper I). This suggests that a long-lasting weak-field disturbances might have the same impact on the total CR depletion as the short-lasting strong-field disturbances. Therefore, two disturbances of the same field strength, but with different durations can cause FDs of different amplitudes, consequently leading to the branching effect in $|F D|$ vs. $B$ plot. This effect could become even more pronounced when geomagnetic activity is included, but may also be masked by it. Strong magnetic fields can 
be characteristics of both strongly geoeffective (strong southern component $B_{\mathrm{S}}$ ) and weakly geoeffective disturbances (weak southern component $B_{\mathrm{s}}$ ). The first one leads to reduced geomagnetic rigidity cutoff and therefore weaker FDs (Chilingarian \& Bostanjyan 2010), so in this case the branching effect would be even more pronounced, whereas in the second case, there would be no additional effect on FD magnitude, so the branching effect would effectively be smeared out by these events.

Because over-recovery represents increased CR flux during/after the recovery phase of Forbush decreases, it might also be related to the change of the geomagnetic rigidity cutoff. Reasoning similar to that regarding masking of FDs caused by reduced geomagnetic cutoff rigidity can be used through competition of Dst index and CR-count recovery-rates. To illustrate that, we sketch in Figs. 9a and b two idealized limiting cases of geoeffective events (full line in FD graphs), compared to nongeoeffective events (dotted line in FD graphs).

In the first case the Dst index is considered to have a very gradual recovery, thus the geomagnetic field is weakened for long time (Fig. 9a). In the second case the Dst index returns to the pre-decrease value rapidly, so the geomagnetic field is affected only for a short time (Fig. 9b). During the Dst depression, i.e., weakened geomagnetic field, low-energy particles can protrude easier, because of the reduced cutoff rigidity. This partly compensates for the CR flux decrease caused by SWD, i.e., the FD amplitude is reduced (i.e., more shallow FD, see, Chilingarian \& Bostanjyan 2009, 2010). If the Dst index remains low during the whole duration of FD, we see a parallel CR flux recovery of geoeffective (full line in Fig. 9a) and nongeoeffective (dotted line in Fig. 9b) event, which results in the CR flux over-recovery of the geoeffective event. On the other hand, if the Dst index returns to the pre-decreased value before FD ends, the FD amplitude would be somewhat reduced, but the recovery of the CR flux would not be greatly affected, and there would be no over-recovery (Fig. 9b).

In reality, one would expect something in between these two schematized cases, where the appearance of the over-recovery is dependent on FD and Dst recovery rates. In Fig. 9c we sketch the scenario by which GMS could cause the over-recovery. As an ICME approaches the Earth, its shielding reduces the CR count rate, i.e., FD starts (denoted as 1 in Fig. 9c). If the ICME has a strong southward magnetic field component, reconnection occurs when the ICME reaches the magnetosphere. The reconnected field lines are transported to the magnetotail, where they reconnect into closed loops. In this process charged particles are energized, and a ring current is produced (dashed curve) through the western-eastern drift that weakens the geomagnetic field (for more details on the formation of the GMS see, e.g., Kane 2005, and references therein). If the geomagnetic field recovers more slowly than the CR count (after the passage of ICME) it will be more permeable for CRs than it was before the event, so an overrecovery is possible (denoted as 2 in Fig. 9c). Especially suitable ICMEs for this scenario would be magnetic clouds whose southward field component is located in their trailing part, i.e., when GMS is delayed.

Finally, the events classified in Sect. 3.3 as N* and $\mathrm{Y}^{*}$ should also be considered. These are events that are either interrupted by another event $\left(\mathrm{N}^{*}\right)$ or start in the recovery phase of another event $\left(\mathrm{Y}^{*}\right) . \mathrm{N}^{*}$ events represent a considerable fraction of the upperbranch population in Fig. 8 and analogously the lower-branch population includes a number of $\mathrm{Y}^{*}$ events.

If we exclude the $\mathrm{Y}$ and $\mathrm{N}$ events from the analysis, we can get an insight into the role of the interruption of an event and over-recovery phenomenon. It follows from the association of

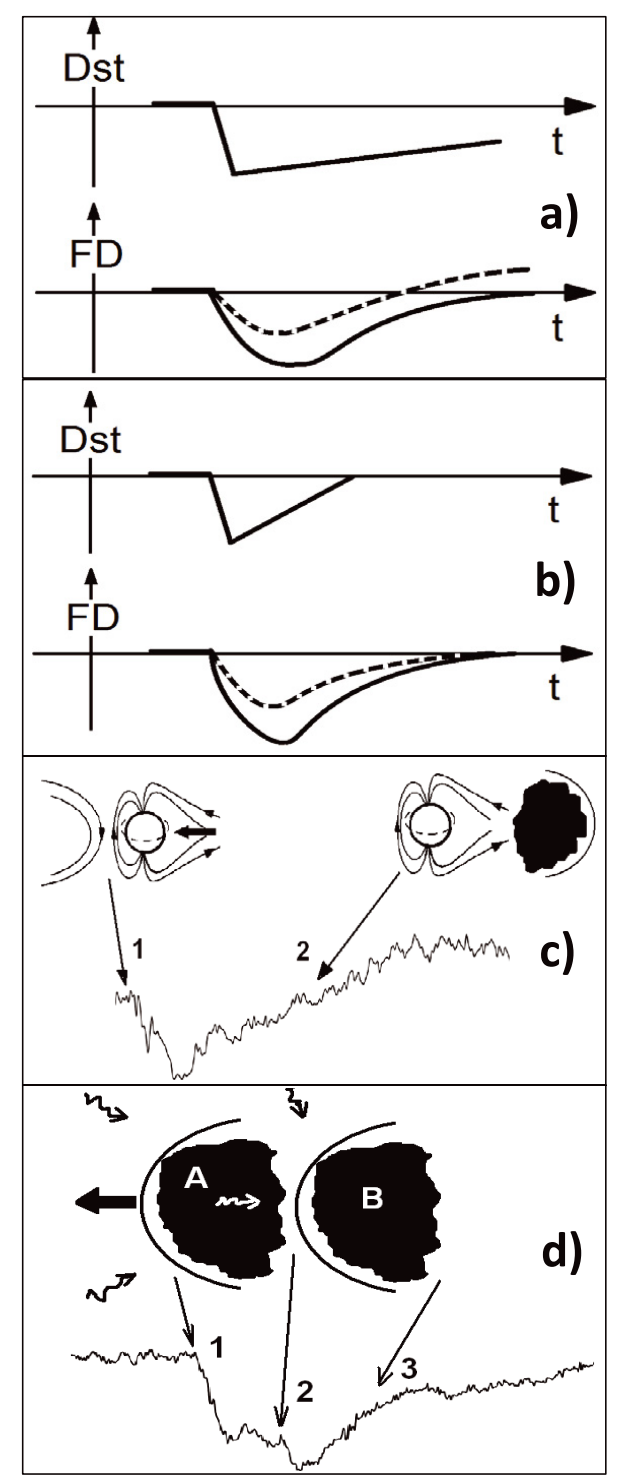

Fig. 9. Different effects of geomagnetic storm on the FD recovery: a) appearance of FD over-recovery as a consequence of Dst slow recovery (dotted line represents FD as it would look like without geomagnetic activity); b) lack of FD over-recovery due to Dst fast recovery; c) two stages of an over-recovery event, where a different CR and Dst recovery rates result in an over-recovery: commencement of FD and GMS (1) and the recovery stage (2); d) two consecutive ICMEs causing two successive depressions, the second depression showing over-recovery.

$\mathrm{N}^{*}$ events with the upper branch and $\mathrm{Y}^{*}$ events with the lower branch that when a new ICME arrives during the recovery period of FD that was caused by some previous disturbance ( $\left.\mathrm{Y}^{*}\right)$, it is likely that the new FD will show an over-recovery (though not all such events were truly over-recovering). Furthermore, to produce the same relative FD depression, the magnetic field of the second ICME must be stronger (branching effect).

This behavior can be explained in simple terms as follows. If one event interrupts the recovery phase of another, one would expect it to show an over-recovery, because the recovery phase of the second event would also include the recovery from the first decrease, resulting in the return of CR count to the pre-decrease level of the first FD. The first ICME eliminates part of CRs, and the second ICME that arrives immediately after reduces additionally the CRs that passed through the first one. However, there 
are now fewer CRs to be eliminated, so a shallower depression is observed. Therefore, to cause the same FD as the first ICME did, the second one should have a stronger magnetic field. This is illustrated in Fig. 9d, where two consecutive ICMEs cause two successive depressions, starting at points denoted in Fig. 9d as 1 and 2. More CRs are available to be depleted when the first ICME arrives, therefore the relative depression in the CR count it causes is deeper. After the passage of both ICMEs, the CR count returns to the pre-event level, resulting in an over-recovery of the second depression (point denoted as 3).

The branching effect therefore might be a combination of several effects:

- Long-lasting weak-field disturbances can cause the same CR depletion as short-lasting strong-field disturbances;

- Two consecutive disturbances do not have the same impact on $\mathrm{CR}$ count;

- Two disturbances of equal field strength are not necessarily equally geoeffective.

Relating the over-recovery phenomenon with reduced cutoff rigidity invokes the question why Jämsén et al. (2007) noted this effect in high-energy muon data, because high-energy CRs are subject to higher cutoff rigidities. As emphasized by (Chilingarian \& Bostanjyan 2009), the decrease of the cutoff rigidity cannot influence the high-energy muon data, because corresponding CRs have more than enough energy to protrude through the terrestrial magnetic field. This indicates that the over-recovery in high-energy muon data and in low-energy neutron monitor data might in fact be two different phenomena.

Acknowledgements. We are grateful to the referee, Dr. Klaus Scherer, whose comments and suggestions led to a substantial improvement in the paper. The research leading to the results presented in this paper has received funding from the European Commission's Seventh Framework Programme (FP7/2007-2013) under the grant agreement no. 218816 (SOTERIA project, www soteria-space. $\mathrm{eu})$.

\section{Appendix A: Monte Carlo analysis}

Owing to the small sample sizes used in Sects. 3.1 and 3.2 the applicability of the $t$-test can become questionable. In order to check the reliability of the $t$-tests, a Monte Carlo test was applied, similarly as in Appendix C of Paper I. Random subsamples of events were generated using our measurements, and random values from an interval defined by measured border-values. Each subsample consisted of the number of the events, defined by our measurements. The correlation coefficient was then calculated for each random subsample, and the procedure was repeated one hundred thousand times. Generally, this provides a normal distribution of correlation coefficients centered around zero. To check the possibility that the correlation coefficient is accidental, we used interval of two standard deviations $(2 \sigma)$, corresponding to the $t$-test value of 0.05 level (i.e., there is only $5 \%$ possibility that the obtained correlation coefficient is accidental). If the correlation coefficient falls in the tail of the normal distribution, i.e. outside the $2 \sigma$ interval, we find it significant, whereas if it falls into the body of the distribution, i.e. within the $2 \sigma$ interval, we find it not significant. This statistical method is less dependant on the sample size than $t$-test, and also enables the detection of outliers, as it leads to the distorted distribution (see Fig. A.1a).

In Fig. A.1 we present an example of the random-sample analysis. To make random samples for calculating correlation coefficient, we used 11 measured $|F D|$ values and generated random $B$ values from the interval $[2,55]$ (note that $2 \mathrm{nT}$ is the weakest measured field, and $55 \mathrm{nT}$ is the strongest measured field).
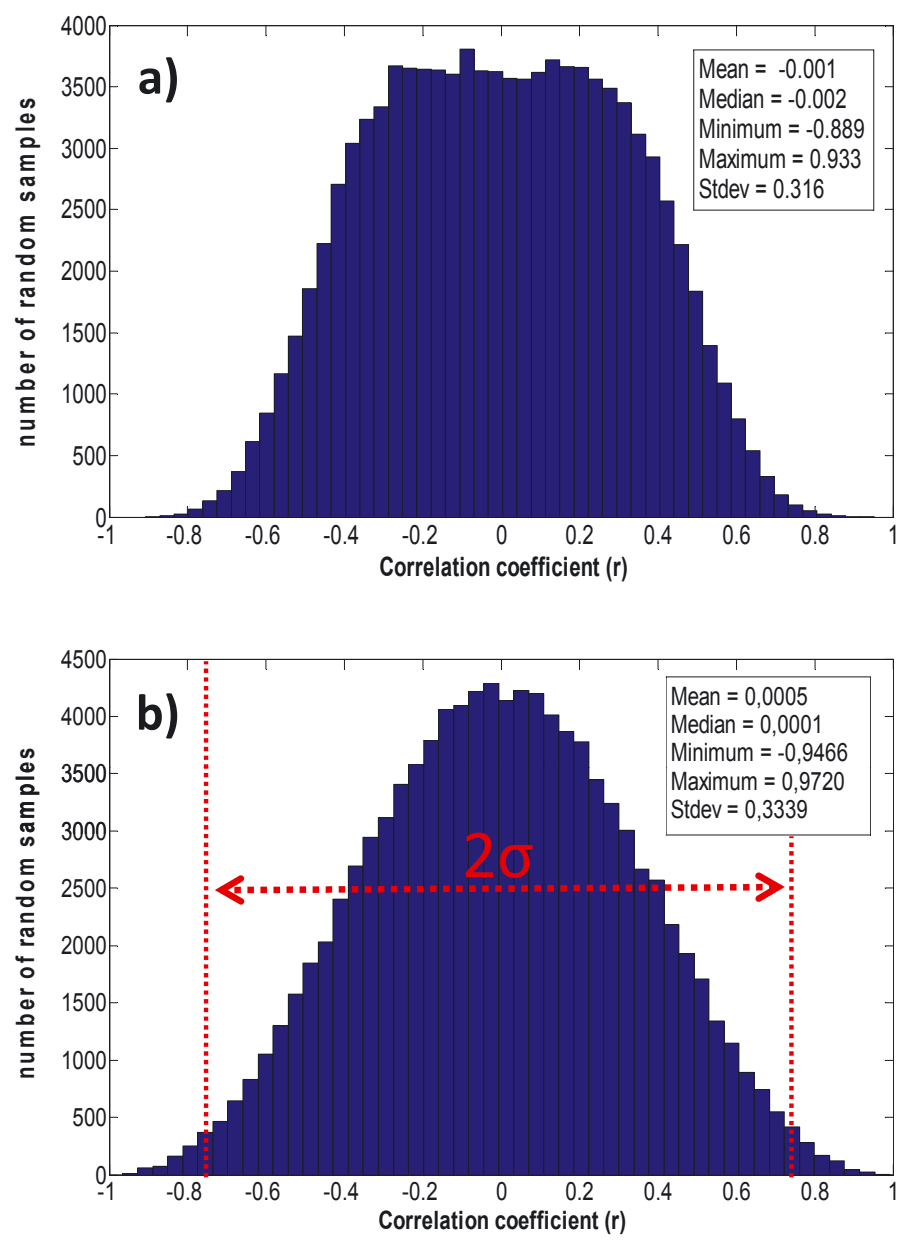

Fig. A.1. Results of the Monte Carlo analysis for the correlation $|F D|(B)$ for mixed events: a) complete sample; b) without the outlier. The $2 \sigma$ interval is marked by red dotted lines.

The result of the Monte Carlo analysis is shown in Fig. A.1a. We see that although the distribution is centered around zero, it shows two peaks, thus deviates from the normal distribution. After removing the outlier, we repeated the procedure with 10 remaining $|F D|$ values and the values of $B$ from the interval [2,24], and obtained the normal distribution of random correlation coefficients (see Fig. A.1b). The corresponding correlation coefficient was found to be $r=0.06$ (see dashed-dotted line in Fig. 5) and this value is located within $2 \sigma$ limit, i.e. there is no statistically significant correlation.

\section{References}

Badruddin. 1996, Ap\&SS, 246, 171

Badruddin, Yadav, R. S., \& Yadav, N. R. 1986, Sol. Phys., 105, 413

Belov, A. V., Eroshenko, E. A., Oleneva, V. A., Struminsky, A. B., \& Yanke, V. G. 2001, Adv. Space Res., 27, 625

Burlaga, L. F., McDonald, F. B., Ness, N. F., et al. 1984, J. Geophys. Res., 89, 6579

Cane, H. V. 2000, Space Sci. Rev., 93, 55

Cane, H. V., Richardson, I. G., \& von Rosenvinge, T. T. 1996, J. Geophys. Res., 101,21561

Chilingarian, A., \& Bostanjyan, N. 2009, J. Geophys. Res. (Space Phys.), 114, 9107

Chilingarian, A., \& Bostanjyan, N. 2010, Adv. Space Res., 45, 614

Dröge, W. 2000, Space Sci. Rev., 93, 121

Dumbović, M., Vršnak, B., Čalogović, J., \& Karlica, M. 2011, A\&A, 531, A91

Forbush, S. E. 1937, Phys. Rev., 51, 1108 
M. Dumbović et al.: Cosmic ray modulation by different types of solar wind disturbances

Iucci, N., Parisi, M., Storini, M., \& Villoresi, G. 1979, Nuovo Cimento C Geophysics Space Physics C, 2, 421

Jämsén, T., Usoskin, I. G., Räihä, T., Sarkamo, J., \& Kovaltsov, G. A. 2007, Adv. Space Res., 40, 342

Kane, R. P. 2005, J. Geophys. Res. (Space Physics), 110, 2213

Koskinen, H. E. J., \& Huttunen, K. E. J. 2006, Space Sci. Rev., 124, 169

Kota, J., \& Jokipii, J. R. 1991, Geophys. Res. Lett., 18, 1797

Kudela, K., \& Brenkus, R. 2004, J. Atmosph. Sol.-Terrest. Phys., 66, 1121

Kuwabara, T., Bieber, J. W., Evenson, P., et al. 2009, J. Geophys. Res. (Space Physics), 114, A05109

Le Roux, J. A., \& Potgieter, M. S. 1991, A\&A, 243, 531

Lockwood, J. A. 1971, Space Sci. Rev., 12, 658

Lockwood, J. A., Webber, W. R., \& Jokipii, J. R. 1986, J. Geophys. Res., 91, 2851

McComas, D. J., Bame, S. J., Barker, P., et al. 1998, Space Sci. Rev., 86, 563

Parker, E. N. 1965, Planet. Space Sci., 13, 9

Penna, R. F. \& Quillen, A. C. 2005, J. Geophys. Res. (Space Physics), 110, 9

Potgieter, M. S. 1998, Space Sci. Rev., 83, 147

Richardson, I. G. 2004, Space Sci. Rev., 111, 267

Richardson, I. G., \& Cane, H. V. 2011, Sol. Phys., 270, 609
Richardson, I. G., Wibberenz, G., \& Cane, H. V. 1996, J. Geophys. Res., 101, 13483

Schwenn, R., dal Lago, A., Huttunen, E., \& Gonzalez, W. D. 2005, ApJ, 23, 1033

Simpson, J. A. 1954, Phys. Rev., 94, 426

Singh, Y. P., \& Badruddin 2006, Sol. Phys., 234, 339

Singh, Y. P., \& Badruddin 2007, J. Geophys. Res. (Space Physics), 112, 2101

Smith, C. W., L'Heureux, J., Ness, N. F., et al. 1998, Space Sci. Rev., 86, 613

Stone, E. C., Frandsen, A. M., Mewaldt, R. A., et al. 1998, Space Sci. Rev., 86, 1

Čalogović, J., Vršnak, B., Temmer, M., \& Veronig, A. M. 2009, in IAU Symp., ed. N. Gopalswamy, \& D. F. Webb, 257, 425

Verkhoglyadova, O. P., Li, G., Zank, G. P., et al. 2010, J. Geophys. Res. (Space Physics), 115, A12103

Vršnak, B., Temmer, M., \& Veronig, A. M. 2007a, Sol. Phys., 240, 315

Vršnak, B., Temmer, M., \& Veronig, A. M. 2007b, Sol. Phys., 240, 331

Wibberenz, G., Le Roux, J. A., Potgieter, M. S., \& Bieber, J. W. 1998, Space Sci. Rev., 83, 309

Yu, X., Lu, H., Le, G., \& Shi, F. 2010, Sol. Phys., 263, 223 